\title{
La compétence numérique dans l'Apprentissage des langues médiatisé par les technologies (ALMT)
}

\author{
Aránzazu Gil Casadomet ${ }^{1}$
}

Recibido: 23 de febrero de 2021 / Aceptado: 14 de julio de 2021

Résumé. Dans cet article, nous présentons une réflexion théorico-méthodologique de l'intégration du Référentiel UNESCO de compétences TIC pour les enseignants (ITC-CFT; 2018, $3^{\mathrm{e}}$ version) et du projet European Digital Competence Framework for Citizens (DigComp; 2017, version 2.1), mené par la Commission européenne, au sein de la Didactique des langues (DDL) et du processus d'enseignement-apprentissage (E/A) d'une langue étrangère, récemment nommé en français l'Apprentissage des Langues Médiatisé par les Technologies (ALMT). Cette réflexion portera notamment sur les différents acteurs de l'environnement numérique dans l'enseignement supérieur, tels que la formation de l'enseignant et de l'étudiant en matière numérique, le contexte de l'environnement, qu'il s'agisse d'un enseignement en présentiel, hybride ou à distance, et la méthodologie de l'enseignement de l'apprentissage dirigé vers l'auto-apprentissage.

Mots-clés: Compétence numérique; langues étrangères; ALMT; DDL; ITC-CFT; DigComp.

\section{[es] La competencia digital en Apprentissage des langues médiatisé par les technologies (ALMT)}

Resumen. En este artículo, presentamos una reflexión teórica y metodológica sobre la integración del Référentiel UNESCO de compétences TIC pour les enseignants (ITC-CFT; 2018, $3^{\mathrm{a}}$ versión) y el proyecto European Digital Competence Framework for Citizens (DigComp; 2017, versión 2.1), liderado por la Comisión Europea, dentro de la Didáctica de las Lenguas (DDL) y el proceso de enseñanza-aprendizaje (E/A) de una lengua extranjera, recientemente denominado en francés Apprentissage des Langues Médiatisé par les Technologies (ALMT). Esta reflexión se centrará en particular en los diferentes actores del entorno digital en la enseñanza superior, como la formación del profesor y del alumno en materia digital, el contexto del entorno, ya sea presencial, híbrido o a distancia, y la metodología de enseñanza del aprendizaje dirigido hacia el aprendizaje autodirigido.

Palabras clave: Competencia digital; lenguas extranjeras; ALMT; DDL; ITC-CFT; DigComp.

\section{[en] Digital competence in Apprentissage des langues médiatisé par les technologies (ALMT)}

Abstract. In this paper, we present a theoretical-methodological reflection on the integration of the UNESCO ICT Competency Framework for Teachers (ITC-CFT; 2018, $3^{\text {rd }}$ version) and of the project European Digital Competence Framework for Citizens (DigComp; 2017, version 2.1), led by the European Commission, into the Didactique des langues (DDL) and the teaching-learning (E/A) process of a foreign language, recently named in French the Apprentissage des Langues Médiatisé par les technologies (ALMT). This reflection will focus in particular on the various actors in the digital environment in higher education, such as the training of the teacher and student in digital matters, the context of the environment, whether face-to-face, hybrid or distance learning, and the methodology of the teaching of directed learning towards self-learning.

Keywords: Digital competence, foreign languages, CALL, DDL, ITC-CFT, DigComp.

Sumario: 1. Introduction. 2. L'Apprentissage des langues médiatisé par les technologies (ALMT). 3. La formation de l'enseignant et de l'étudiant en matière numérique. 4. Le contexte de l'environnement numérique: du présentiel à la méthodologie à distance. 5. La méthodologie de l'enseignement: de l'apprentissage dirigé à l'auto-apprentissage. 6. En guise de conclusion. 7. Liste des abréviations. 8. Bibliographie.

Cómo citar: Gil Casadomet, Aránzazu (2021). La compétence numérique dans l'Apprentissage des langues médiatisé par les technologies (ALMT). Didáctica. Lengua y Literatura, 33, 133-144. 


\section{INTRODUCTION}

La formation en langues a évolué au cours du XXI ${ }^{\mathrm{e}}$ siècle vers un contexte numérique devenant essentiel pour le développement personnel, professionnel et culturel de la société. L'incorporation massive de ressources technologiques dans les salles de classe a donné lieu à un foisonnement de réflexions sur la compétence numérique et l'enseignement de langues étrangères, notamment depuis l'incontournable situation actuelle que nous traversons. Il est ainsi nécessaire de continuer à étudier et à réfléchir sur leur adéquation et leur mise en œuvre dans une société en constante évolution technologique. En ce sens, le projet European Digital Competence Framework for Citizens (DigComp v.2, 2016), mené par la Commission européenne, cherche à unifier les composantes des compétences numériques pour le travail, l'apprentissage, le temps libre, l'inclusion, la participation dans la société, entre autres. Ce cadre a été publié pour la première fois en 2013 par la Commission européenne et il a offert une référence commune au niveau européen quant à la manière d'identifier et de décrire les domaines clés de la compétence numérique, qui est comprise comme une utilisation confiante et critique des outils technologiques de l'information et de la communication (TIC) dans ces domaines (Vuorikari, 2016, p. 5). De 2013 à la publication en 2016 de sa deuxième version, DigComp a été utilisé parallèlement à la numérisation rapide de divers aspects de la société qui pose de nouvelles exigences, d'où la nécessité de la version 2.0. Tout au long du parcours de ce cadre, nous pouvons distinguer un nouveau concept des technologies, à savoir que les appareils technologiques sont devenus une partie intégrante de la communication courante, face au concept précédent où les technologies étaient envisagées comme un ajout à cette communication créant un contexte particulier (Kern, 2010).

Compte tenu de ces considérations, nous nous interrogeons à maintes reprises sur la manière dont s'intègre ce cadre à la Didactique des Langues (DDL; André, 1989) et au processus d'enseignement-apprentissage (E/A) d'une langue étrangère, ou tel qu'il est récemment nommé en français, l'Apprentissage des Langues Médiatisé par les Technologies (ALMT; Guichon, 2012a). C'est pour cette raison que nous avons l'intention d'analyser l'usage des technologies pour la formation en langues dans l'enseignement supérieur, usage qui implique un déploiement mobilisant plusieurs acteurs, en raison de leur assignation, selon (1) la formation de l'enseignant et de l'étudiant en matière numérique, lesdits natifs ou immigrants en technologie (Prensky, 2001), (2) le contexte de l'environnement, soit présentiel, hybride ou à distance et (3) la méthodologie de l'enseignement de l'apprentissage orienté vers l'auto-apprentissage.

\section{L'APPRENTISSAGE DES LANGUES MÉDIATISÉ PAR LES TECHNOLOGIES (ALMT)}

Les origines de cette discipline sont liées au développement de l'Enseignement Assisté par Ordinateur (EAO; Demaizière, 1986) et de l'Acquisition ou Apprentissage des Langues Assisté par Ordinateur (ALAO; Chanier, 1995; Mangenot, 2000), acronyme francisé de Computer-Assisted Language Learning (CALL; Lévy, 1997). ${ }^{2}$ Guichon (2012b) explique comment le concept vis-à-vis des compétences numériques dans l'acquisition des langues a changé, donnant lieu aussi à un changement du nom de la discipline tout au long de ces quarante dernières années:

Tout d'abord, médiatisé par les technologies semble plus adapté qu'assisté par ordinateur car la première formulation permet de prendre en compte une évolution des objets de recherche. En effet, si l'ordinateur fut longtemps l'objet pivot de la réflexion scientifique, avec l'arrivée d'outils comme les MP3 ou les smartphones, puis l'avènement d'Internet et son développement, ce sont les technologies au sens large qui ont complexifié le paysage de la recherche, ce qui justifie de ne plus s'intéresser seulement à l'ordinateur mais à son hyperonyme technologies. L'appellation Apprentissage des langues médiatisé par les technologies revendiqué ici permet ainsi de ne plus se centrer sur le seul ordinateur en ce qu'il donne accès et organise l'information mais d'inclure les utilisateurs, les usages, les conventions pour communiquer. Cela permet une distinction entre la technique et la technologie, celle-ci étant définie par Dieuzeide (1994: 12) comme l'étude de l'emploi des outils, appareils, machines, matériaux en vue d'une action définie et l'étude de leurs effets. Il s'agit, dès lors, non plus seulement de considérer ce que l'outil offre comme possibilités à l'apprentissage d'une langue mais de prendre en compte les différents aspects qui reconfigurent, au moins en partie, les actes d'enseigner et d'apprendre une langue et en font des événements cognitifs mais aussi sociaux qui dépassent le simple fait de se dérouler par le biais d'ordinateurs. (Guichon, 2012b, p. 5)

L'objectif de légitimer ce champ de recherche en didactique des langues étrangères est aussi de relever les traces historiques et les grandes étapes de la constitution d'un collectif, ainsi que de repérer des intérêts communs aussi bien autour des pratiques d'enseignement que de la recherche (Soubrié, 2020).

Plusieurs auteurs (Grosbois, 2012; Guichon, 2012a; Albero, 2013; Soubrié, 2020) font le point récemment sur une rétrospective du numérique et les potentialités qu'il représente pour l'apprentissage des langues. Que ce soit un outil

Cf. Trujillo Sáez et al. (2019) et Gabarrón Pérez et al. (2020) pour une révision de la littérature concernant le Computer-Assisted Language Learning (CALL). 
pour apprendre avec le numérique, ou un environnement pour apprendre dans le numérique, celui-ci «est également envisagé comme un lieu d'échange et de socialisation. Les technologies ne viennent plus alors seulement soutenir l'enseignement-apprentissage, mais permettent également d'immerger les apprenants dans un bain linguistique et culturel» (Soubrié, 2020, p. 32). La médiatisation est donc véhiculée par le fait social intégré dans la communication langagière et le numérique en fait partie par extension. La communication est primairement déterminée par la relation interpersonnelle et l'interaction sociale intersubjective (Ollivier et al., 2018), comme une ouverture sur le monde, c'est-à-dire «du réel» en classe de langues étrangères, c'est ce que le numérique permet de réaliser en ligne (Albero, 2010). La communication médiatisée par ordinateur (CMO, terme francisé de Computer-mediated communication $(\mathrm{CMC})^{3}$ for Language learning and teaching (CMCL; Lamy et Hampel, 2007) dans l'enseignement, intègre la compétence numérique moyennant la réalisation des objectifs langagiers et communicationnels (Guichon, 2012a). En ce sens, l'importance des ressources utilisées par les étudiants en contexte plurilingue et multimodal pour exploiter les atouts de nouvelles technologies est évoquée à plusieurs reprises (Mangenot, 2011, 2013; Massou, 2017; Schaeffer-Lacroix, 2020).

\section{LA FORMATION DE L'ENSEIGNANT ET DE L'ÉTUDIANT EN MATIÈRE NUMÉRIQUE}

L’UNESCO a élaboré un Référentiel de compétences TIC pour les enseignants (ICT-CFT; 2008, version 3 en 2018) avec l'intention de repenser le rôle des enseignants par rapport à l'avenir de l'enseignement et l'application des TIC ainsi que pour aider les pays à développer des politiques et des normes nationales complètes concernant les compétences TIC des enseignants et l'intégration des TIC dans tous les plans d'enseignement. Dans un premier stade, ce référentiel comprend l'alphabétisation technologique comme une approche pour préparer les apprenants, les citoyens et la population active à se servir des TICE (TIC + Enseignement) pour favoriser le développement social et améliorer la productivité économique. De la même manière, les enseignants doivent «être capables d'utiliser les TIC pour gérer les données relatives à leur classe et assurer leur propre formation professionnelle» (UNESCO, 2012, 11). En définitive, ces acteurs doivent utiliser des ordinateurs et des logiciels de productivité, des logiciels d'entraînement, des tutoriels et des contenus Web, ainsi que des réseaux pour des tâches de gestion. Dans la troisième version du référentiel, ce premier stade est défini comme «l'Acquisition de connaissances, auquel les enseignants acquièrent des connaissances sur l'usage qu'ils peuvent faire des technologies et des compétences de base dans le domaine des TIC» (UNESCO, 2018, p. 8).

Cette approche est élargie à tout domaine de l'enseignement, également à celui des langues étrangères et au niveau supérieur, car l'enseignant doit acquérir de nouvelles compétences professionnelles s'il veut s'adapter à la nouvelle ère du numérique. Trujillo Sáez et Raso Sánchez (2010) estiment que:

La integración metodológica de las TIC ha de propiciar la constitución de este nuevo entorno, donde la Universidad de hoy no permanezca anclada en valores y formas del pasado, sino que se conforme como principio de esperanza para responder a las nuevas necesidades en un marco contextual social que presenta Internet como principio de apoyo y espacio natural. [...] El nuevo perfil del docente del siglo xxI aboga por el cambio metodológico en el que prime una adecuada alfabetización digital que incite consecuentemente la renovación didáctico-organizativa de los procesos de enseñanza-aprendizaje. Para ello, mejor y mayor cualificación profesional inicial. [...] La integración TIC y el uso de herramientas web 2.0 en los procesos de enseñanza-aprendizaje han facilitado la colaboración, el trabajo en equipo, el diálogo e intercambio efectivo de ideas y la experiencia contextualizada y significativa de los futuros docentes. (Trujillo Sáez et Raso Sánchez, 2010, pp. 58-74)

Cependant, leur étude révèle que dans le domaine de l'enseignement supérieur:

No se realizan cursos formales de aprendizaje en torno a las TIC. La formación principalmente surge de la iniciativa propia y de la navegación y búsqueda en la red. Sin embargo, se reclaman mayores actividades formativas en cuanto a la integración TIC en la Universidad. De igual modo, existe un déficit de conocimiento y uso de web de recursos educativos de calidad contrastada para formación permanente del profesorado. [...] Se echa en falta una mayor praxis en la Universidad en cuanto a la integración y uso de las TIC. Se reclama mayor presencia de actividades que promuevan este proceso de alfabetización digital. (Trujillo Sáez et Raso Sánchez, 2010, p. 75)

Cette acquisition implique un effort puisqu'il faut briser les structures mentales pour s'adapter à une nouvelle façon d'enseigner et d'apprendre. Muñoz-Moreno et Peirats (2016) ou Pérez Cervantes et Francisco Saker (2016) sont également en accord avec ces déclarations tout en considérant que les TICE deviennent des outils indispensables dans les établissements d'enseignement supérieur, car elles permettent l'échange d'informations diverses, de traite-

Cf. Kern (2006), pour une connaissance des origines et une rétrospective de la communication médiatisée par ordinateur en langues du ComputerMediated Communication (CMC). 
ments de texte, de pages web interactives pour l'apprentissage, de présentations multimédia, etc. Les technologies de l'information et de la communication permettent des interactions entre les étudiants eux-mêmes et avec l'enseignant, favorisant l'attitude active et la réflexion critique. Elles offrent également les outils et les connaissances nécessaires à l'accomplissement des tâches, augmentent la participation et développent l'esprit d'initiative chez les étudiants, ce qui leur permet de filtrer l'information, de sélectionner et de prendre des décisions. Ainsi, le numérique peut être un moteur de rénovation pédagogique et un déclencheur de réflexions fructueuses sur les pratiques enseignantes (Ollivier et Puren, 2013).

Prensky (2001) a établi une distinction entre les natifs numériques (ceux qui sont nés à l'ère des TIC) et les immigrants numériques (ceux qui sont nés avant l'existence de ces réseaux et supports et qui ont besoin d'une période d'apprentissage et d'adaptation à l'âge adulte):

What should we call these "new" students of today? Some refer to them as the N-[for Net]-gen or D-[for digital]-gen. But the most useful designation I have found for them is Digital Natives. Our students today are all "native speakers" of the digital language of computers, video games and the Internet. So what does that make the rest of us? Those of us who were not born into the digital world but have, at some later point in our lives, become fascinated by and adopted many or most aspects of the new technology are, and always will be compared to them, Digital Immigrants. (Prensky, 2001, p. 1)

La nécessité de former les étudiants universitaires (Area, 2010; Gisbert et Esteve, 2011), aussi bien les natifs que les immigrants numériques, prévaut dans les compétences numériques, car ils doivent pouvoir accéder à de nouvelles informations, développer des compétences de recherche, développer leur capacité à construire des connaissances par l'interaction, savoir s'exprimer et communiquer avec de nouveaux langages et outils et, enfin, acquérir des compétences pour apprendre dans des espaces enrichis et construits avec les technologies.

Dans un deuxième stade de l'ICT-CFT, la mise en œuvre de l'alphabétisation de l'information et des données et de la création de contenus numériques ainsi que l'acquisition des connaissances dans des environnements d'E/A des langues s'avère très utile pour l'approfondissement des connaissances, la communication et le travail collaboratif (UNESCO, 2008). Dans le cadre de l'Espace européen de l'enseignement supérieur (EEES), l'importance que représente l'exigence de l'acquisition de certaines compétences chez les enseignants et les étudiants est manifeste. Les compétences à développer par les professeurs d'université vont actuellement au-delà d'une instruction, il s'agit de parvenir à une amélioration significative de la qualité du processus d'enseignement-apprentissage de l'étudiant. La réforme du processus de Bologne (2009), concernant les institutions de l'enseignement supérieur des 48 États membres de l'EEES, implique une mise à jour à la fois méthodologique et conceptuelle. Ce cadre éducatif exige que les enseignants s'impliquent non seulement dans l'apprentissage, mais aussi dans le développement intégral des étudiants, qu'ils soient prêts à changer et possèdent un ensemble de compétences qui sont parfois sous-développées. Certaines perspectives pour 2020 étaient de «mieux prendre en compte la dimension sociale corrélée à l'éducation et à la formation tout au long de la vie» ou de «favoriser un enseignement conçu selon une approche centrée sur les connaissances et les compétences, ainsi que l'insertion professionnelle des étudiants à 1'issue de leur formation ${ }^{4}$.

Demaizière et Grosbois (2014) et Grosbois (2015) suggèrent que le numérique permet de construire des scénarios aidant à la production langagière, par exemple à travers les échanges grâce à la visioconférence. Ces échanges favorisent la capacité de gérer l'information, structurer les tâches à réaliser et intégrer des outils logiciels dans des projets en collaboration. Elle est pertinente en formation de langues étrangères, étant donné qu'elle permet, pour les enseignants, un changement de paradigme progressif orienté vers un programme universitaire combinant la présence et l'apprentissage virtuel ou l'apprentissage mixte et favorisant un apprentissage significatif ainsi que le développement de compétences communicatives et numériques.

Comme troisième et dernier stade de l'ICT-CFT, l'approche création de connaissances est présent lors de l'activation de certaines compétences telles que «l'aptitude à résoudre des problèmes, à communiquer, à travailler en collaboration, à faire des expérimentations, à exercer son esprit critique et à faire preuve de créativité» (UNESCO, 2012, p. 15). La classe de langues étrangères devient une communauté d'apprentissage dans laquelle le développement des compétences d'apprentissage mutuelles des étudiants est fomenté. De plus, une grande diversité de ressources numériques et d'environnements électroniques est mise à disposition pour créer cette communauté et l'aider à produire des savoirs linguistico-culturels. Puren (2013) constate que la typologie de ressources disponibles en Didactique des Langues-Cultures (DLC) favorise cette création de connaissances en classe de langues étrangères et pour le processus d'E/A:

Les ressources technologiques [...] sont actuellement, depuis l'avènement de l'ère informatique, d'une très grande richesse, puissance et souplesse [...]. Les potentialités de ces ressources technologiques sont énormes, tant pour l'éducation en général que pour l'enseignement-apprentissage des langues, et elles représentent d'ailleurs depuis des années une source d'innombrables expérimentations innovantes que le flux constant

4 Lien de consultation: https://www.enseignementsup-recherche.gouv.fr/cid56043/presentation-de-1-e.e.e.s.html\#Bologne-2020 
des évolutions technologiques vient réalimenter en permanence. Ce stade expérimental ne peut être dépassé, cependant - en d'autres termes, l'innovation ne peut se diffuser et s'enraciner -, que si, dans des espaces plus larges que les seuls terrains d'expérimentation et des temps plus longs que ceux nécessaires à écrire un compte rendu de recherche, parviennent à s'établir et à se maintenir des relations stables et durables entre ces ressources technologiques et les autres. (Puren, 2013, p. 4)

En 2013, la Commission européenne actualise l'ICT-CFT et mise sur l'identification et la description des composantes clés de la compétence numérique en termes de connaissances, habilités et aptitudes. Par ailleurs, les citoyens ont la nécessité de recourir à cette compétence et aux moyens numériques pour accomplir des tâches, résoudre des problèmes, communiquer, gérer de l'information, collaborer, créer et partager des contenus pour créer des connaissances d'une façon efficace, adéquate, éthique, précise, etc. Il s'agit d'un projet qui reçoit le nom d'European Digital Competence Framework for Citizens (DigComp) et que les pays européens adoptent et adaptent pour leurs citoyens en matière de travail, divertissement, apprentissage, consommation, évolution, etc. En 2016, la deuxième version des résultats du projet est publiée et certains domaines d'action y sont approfondis. Ils sont regroupés en 5 niveaux: l'information et l'alphabétisation des données, la communication et la collaboration, la production de contenu numérique, la sécurité et la résolution de problèmes. Ces domaines sont clés comme objectif de planification pédagogique, étant donnée l'importance concédée à l'éducation des adultes:

Professional development programmes for teachers in various EU countries have embraced the DigComp Framework for teachers' digital competence building. For example, the Ministry of Education, Culture and Sports in Spain created A Common Framework for Digital Competence for Teachers (Marco común de Competencia Digital Docente 2.0) in 2014. Since then, INTEF develops new digital training materials for teachers based on DigComp. These include, for example, a Massive Open Online Course (MOOC) on how to teach and evaluate digital competence, already in its $3^{\text {rd }}$ edition. In 2016, a number of short, 3 hours per unit, online courses on DigComp were also launched. Moreover, the use of DigComp for Professional Development (PD) for teachers has been agreed between the state and regional governments. This has encouraged implementation, e.g. Extremadura has introduced a digital competence portfolio for teachers based on the Spanish model of Teacher DigComp. (Vuorikari et al., 2016, p. 21)

La dernière version du DigComp (v. 2.1; Carretero, Vuorikari et Punie, 2017) et le projet parallèle DigCompEdu, ${ }^{5}$ rendus publics en 2017, fournissent des exemples à propos des compétences numériques qu'il faut acquérir dans l'enseignement; elles sont classifiées en huit niveaux avec deux sous-niveaux pour chacun (fondation, intermédiaire, avancé et expert). Ainsi, tout comme d'autres cadres de référence européens concernant l'enseignement des langues étrangères comme par exemple, le Cadre européen commun de référence pour les langues (CECR) ou le Cadre de référence pour les approches plurielles des Langues et des Cultures (CARAP), ${ }^{6}$ les apprenants des compétences peuvent suivre la progression de leur acquisition pas à pas. Parmi les différentes compétences numériques du DigComp v.2.1, il est intéressant de citer ici les suivantes pour prendre conscience de leur application dans le domaine de l'enseignement:

- Mener une recherche d'information sur un support numérique pour se tenir au courant de l'actualité d'un sujet.

- Stocker et organiser des données dans différents types d'espaces de stockage (ordinateur, disque dur externe, environnements numériques sécurisés, etc.).

- Utiliser un dispositif approprié pour partager des contenus et connaître les bases fondamentales de la propriété intellectuelle.

- Produire et modifier des documents à contenu textuel et multimédia à l'aide de différentes applications dans une perspective de partage et apprentissage des savoirs.

- Choisir et appliquer des mesures de protection de l'environnement informatique, des données personnelles et de la vie privée lors des navigations en ligne.

\section{LE CONTEXTE DE L'ENVIRONNEMENT NUMÉRIQUE: DU PRÉSENTIEL À LA MÉTHODOLOGIE À DISTANCE}

Très longtemps répandu, l'enseignement en présentiel a été le cadre de référence pour le processus d'E/A. Cette méthodologie réduit toutes les possibilités de mener l'interaction complète de l'enseignement des langues étrangères sauf si «l'enseignement s'effectue de façon 'hybride'» (blended learning), c'est-à-dire «qu'il conjugue la communi-

\footnotetext{
Lien de consultation: https://ec.europa.eu/jrc/en/publication/eur-scientific-and-technical-research-reports/european-framework-digital-competenceeducators-digcompedu

6 Il faut noter que le CECR $(2001,2005)$ et le CARAP (2008) intègrent très pauvrement l'usage des nouvelles technologies dans l'enseignement des langues étrangères. Le volume complémentaire du CECR paru en 2018 suit la même caractéristique de conception par laquelle les compétences numériques se trouvent hors les compétences d'acquisition parmi les langagières, communicatives et culturelles.
} 
cation en présentiel et la communication en ligne» (Develotte, 2010, p. 455), grâce à la médiatisation des technologies et la conception d'environnements numériques d'apprentissage (ENA).

La méthodologie à distance n'est pas une invention nouvelle, cependant elle a acquis une certaine importance au sein de l'enseignement grâce aux bénéfices de la praticité et du guidage des enseignants. D'après Beacco et Luzzati (1999), il est convenable de pouvoir disposer de:

- Accompagnement de l'apprentissage autonome par des indications intégrées au matériel: indications de parcours privilégiés, diagnostic d'erreur et renvoi vers des activités de systématisation destinées à améliorer les compétences de l'apprenant...

- Recours à des aides en ligne comme la traduction (de mots, de consignes, de documents servant de support aux activités, des informations métalinguistiques ou culturelles...), la version orale de documents écrits, la transcription de documents sonores, l'accès à un dictionnaire bilingue ou monolingue (ou à un lexique)...

- Possibilité d'interactions différées avec un tuteur: messages de demande d'informations, correction personnelle des activités, envoi de fichiers son ou textes relatifs aux activités, pour mise au point et commentaire par le tuteur.

- Interaction en temps réel avec un tuteur, nouvelle forme du présentiel dans un enseignement individualisé.

- Interaction en ligne ou différée avec d'autres apprenants impliqués au même moment dans le même matériel ou la même séquence, dans le cadre d'une formation mutuelle ou coopérative. (Beacco et Luzzati, 1999, p. 4)

Dans le cas de la formation à distance, Beacco et al. (2000) et Mangenot (2003) suggèrent que pour l'accomplir il est nécessaire d'adapter le matériel didactique aux besoins langagiers des apprenants. Par ailleurs, les ressources numériques (plateformes de visioconférence, Internet, apps, etc.) privilégient certaines compétences d'interaction et production écrite et d'interrelation (écrite et orale). Outre le matériel didactique, le guidage des enseignants semble important et l'étudiant doit être assisté en permanence. Cette aide pourra être réglée grâce aux multiples dispositifs de communication mis à la disposition de l'étudiant dans l'enseignement supérieur (visioconférence, chat, appel téléphonique, messagerie, etc.). Lebrun (2011), Fernández et Paricio (2019), Cabero Almenara (2019), entre autres, sont en accord avec l'idée que les plateformes LMS (Learning Management Systems), telles que WebCT ou Moodle, ont constitué un excellent progrès à cet égard, en facilitant l'accès à ce matériel. Un environnement d'apprentissage est lié à tout ce qui entoure le processus d'apprentissage des personnes en général et des enseignants en particulier. Il peut s'agir d'activités, d'outils, d'espaces, etc. De surcroît, les étudiants accordent une grande valeur à ces supports technologiques accessibles (Guichon, 2015; Abadía Valle et al., 2015).

La relation entre les ENA et l'utilisation quotidienne des appareils numériques est présente dans notre société. Le DigComp 2.1 (2017) conçoit comme une des compétences numériques l'insertion dans le monde numérique pour maîtriser les stratégies et les enjeux de la présence en ligne lors des processus d'enseignement-apprentissage. Cette compétence encourage le citoyen à se positionner en tant qu'acteur social dans le monde numérique, en lien avec les règles et les valeurs de la présence publique sur le web, les environnements numériques, les réseaux sociaux, etc. En ce sens, l'utilisation quotidienne des appareils numériques favorise le changement des routines et des habitudes des étudiants (Soubrié, 2020) et constitue une forme d'accès à l'information. Ces scénarios influencent le système universitaire, à savoir le développement rapide des TIC appliquées à l'enseignement en général et des langues en particulier. Par conséquent, une impulsion à l'enseignement en ligne s'ouvre avec un regard critique qui permet de réfléchir aux nouveaux défis demandés par la société contemporaine (Bates, 2001; Mangenot, 2011; Rodríguez Correa et González Sanmamed, 2013).

Une forte variable qui concerne particulièrement l'enseignement en ligne est la temporalité de la communication, puisque celle-ci peut être diffusée entre les enseignants et les étudiants de façon synchrone ou asynchrone. La communication synchrone permet à deux ou plusieurs personnes de dialoguer simultanément, en temps réel, par l'intermédiaire d'un environnement numérique, sans oublier que pour qu'elle soit synchrone, il faut que l'orateur et l'auditeur coïncident dans le temps, ce qui n'a pas nécessairement lieu dans la communication asynchrone (Montero Curiel, 2019). Cette variable communicative influe sur le type d'apprentissage:

le travail mettant en jeu la réflexion, le traitement d'une information complexe ou la métacognition sera davantage facilité par le monde asynchrone tandis que les aspects socioaffectifs, interactionnels et liés au développement de la fluidité seront plutôt favorisés par le mode synchrone. (Guichon, 2012a, p. 161)

L'ALMT asynchrone permet d'ailleurs de potentialiser les bénéfices des échanges électroniques sur le plan linguistique et aussi culturel (Kern, 2006). Des échanges où l'apprentissage interculturel prend sa place dans le cadre de l'acquisition des langues étrangères, la culture et la compétence interculturelle. ${ }^{7}$ L'ALMT synchrone, à son tour, encourage les apprenants à collaborer et participer activement tout en réduisant l'anxiété associée à l'expression orale en langue étrangère.

Dans le cadre de la Didactique des Langues-Cultures (DLC), Puren (2019, p. 219) explique le fait positif que produit 1'acquisition de cette compétence, composante de la compétence culturelle, dans le sens «d'être capable de prendre de la distance par rapport à sa propre culture et d'être attentif aux incompréhensions et interprétations erronées toujours possibles entre personnes de cultures différentes». 
À l'aide du tableau élaboré par Guichon (2012a, p. 157), les ENA, selon les différents modes de communication médiatisée par ordinateur à des fins didactiques en mode asynchrone et textuel, peuvent être le courrier électronique, le forum, les blogs, les wikis, les chats, etc.; et en mode audiovisuel, les réseaux sociaux de partage de clips sonores ou vidéos (podcasts; Facebook, Twitter, YouTube), les plateformes LMS (WebCT, Moodle, Canvas), etc.; en mode synchrone, ils peuvent être la messagerie instantanée (Whatsapp), la visioconférence (Skype, Teams, Google Meet, Zoom), les mondes virtuels (jeux vidéo), etc. Toute cette prolifération d'environnements est due à l'impact médiatique et social des supports électroniques tels que les ordinateurs, les tablettes, les phablettes, les smartphones, les télévisions, etc. (Barroso Osuna, 2019).

\section{LA MÉTHODOLOGIE DE L'ENSEIGNEMENT: DE L'APPRENTISSAGE DIRIGÉ À L'AUTO- APPRENTISSAGE}

C'est au milieu du XVIIe siècle que nous observons une indication de l'importance que les langues modernes commencent à prendre dans l'enseignement des langues. Puren (2012) explique à cet égard que la langue latine était enseignée à cette époque-là à partir de la langue maternelle, et l'apprentissage dirigé passait de l'étude directe des textes classiques à des fragments adaptés avec leur traduction dans la langue maternelle. Cette méthodologie d'enseignement, dite «traditionnelle» sera plus tard appelée «grammaire-traduction», un système qui consiste en un exercice de mémoire guidé. C'est avec la méthodologie audiovisuelle, trois siècles plus tard, que l'accompagnement de l'enseignant vise à être contextualisé par des supports sonores et visuels l'assimilation du vocabulaire et de ses significations (Delgado, 2003). En définitive, il s'agissait de guider les apprenants pour réaliser des études lexicales et grammaticales, pour rendre l'apprentissage des langues aussi simple que possible et pour faire de ce qui était appris une connaissance à laquelle il est possible d'avoir recours:

La méthodologie audiovisuelle a l'avantage de prendre en compte le contexte social dans lequel la langue est utilisée. Ainsi, cela permet aux apprenants d'apprendre relativement rapidement à communiquer oralement avec des natifs, bien que les dialogues soient assez limités. (Pérès, 2013, p. 16)

L'apprentissage des langues ne sera plus une simple formation d'habitudes acquises par la répétition ni une collection de règles grammaticales ou de mots hors contexte. L'objectif principal sera d'apprendre à l'étudiant à savoir comment communiquer.

Le passage de l'apprentissage guidé à l'auto-apprentissage aura lieu à la fin du Xx $x^{e}$ siècle. André (1989) faisait déjà référence au mot autonomie dans l'apprentissage des langues et la Didactique des Langues (DDL). Ses collègues Moulden (1989) ou Holec (1989a et 1989b), dont les publications sont incluses dans l'ouvrage d'André, font aussi référence aux concepts d'apprentissage autodirigé et d'autonomisation, tous les deux à mi-chemin entre l'apprentissage dirigé et l'autonomie en formation. Ces variantes comprennent à leur tour différentes méthodologies de travail, telles que le travail autonome individuel fait à la maison, le travail autonome en petit groupe fait sur place ou à la maison et le travail autonome individuel ou de petit groupe effectué sur place «en atelier». Pour autant, l'autonomie est une «capacité [qui] n'est pas innée, elle doit s'acquérir, soit de manière naturelle, soit par apprentissage formel» (Holec, 1989a, p. 31) et elle est donc opposée à la notion de dépendance (Barbot, 2001; Tremblay, 2003; Albero, 2013). Ainsi tout comme l'explique Albero (2003):

S'intéresser à la notion d'autoformation en ces termes aujourd'hui, c'est se préoccuper du devenir politique du système éducatif lié aux cycles du supérieur, à celui de la construction d'un espace social où l'utilitarisme ne l'emporte pas toujours sur la dimension humaniste et la productivité sur la dimension culturelle et poḯtique de l'activité humaine. C'est également se préoccuper de la qualité de la formation impartie aux personnes, surtout en une période où la puissance des technologies de l'information et de la communication peuvent si rapidement influer les systèmes de pensée au niveau planétaire. (Albero, 2003, p. 67)

Dans ce contexte, le DigComp 2.1 (2017) signale plusieurs compétences numériques propres à l'autoformation, par exemple organiser et optimiser des contenus numériques pour les utiliser en ligne ou interagir et communiquer avec des individus et de petits groupes par messagerie, système de visioconférence, etc., c'est-à-dire utiliser différents outils ou services de communication numérique. Les étudiants prennent en charge leur propre apprentissage sous forme de dispositifs numériques, entre autres, du type SPOC (Small private online course), SPOOC (Self-Paced Open Online Course), MOOC (Massive open online course) ainsi que leurs variantes xMOOC, cMOOC ou tMOOC, ${ }^{8}$ voire LMOOC pour l'enseignement des langues, ou CLOM (Cours en ligne ouverts et massifs) selon la terminolo-

«Los XMOOC tienden a ser cursos universitarios tradicionales de e-learning que se adaptan a las características de las plataformas de los MOOC, mientras que los cMOOC se apoyan en la filosofía del aprendizaje conectivista, y los tMOOC se apoyan en la teoría constructivista y se centran en que los estudiantes realicen diferentes tareas y actividades sobre la información ofrecida» (Cabero Almenara, 2019, p. 84). 
gie en français, et NOOC (Nano Open Online Massive). ${ }^{9}$ En définitive, des cours d'enseignement de connaissances dont les différentes caractéristiques oscillent entre la méthodologie d'apprentissage, la temporalité, la quantité de contenus, la gratuité ou le prix et la concession d'un certificat de réussite, entre autres, et dont le guidage asynchrone permet à l'étudiant de développer son autonomie sans la présence directe de l'enseignant (Rivens Mompean et Eisenbeis, 2009), mais sans que la substitution de celui-ci soit totale (Mangenot, 2017). Nous sommes donc en accord avec Linard (2003, p. 246) pour dire qu' «Autoformation et TIC requérant également l'autonomie, elles devraient s'allier sans peine dans des solutions efficaces, en formation en distance en particulier».

\section{EN GUISE DE CONCLUSION}

Notre réflexion portée sur l'ALMT présente un domaine de recherche à l'intérieur de la didactique des langues et tient compte des compétences numériques postulées par certains cadres référentiels comme l'ICT-CFT de l'UNES$\mathrm{CO}$ ou le DigComp de la Commission européenne. Il s'agit donc d'immerger les enseignants et les étudiants dans un bain linguistique et culturel et les sensibiliser à des formes particulières de communication grâce à des référentiels de compétences numériques guidant les citoyens. Ces cadres de référence plongent les étudiants dans la «vie réelle», moyennant des ENA, en multimodalité et plurilinguisme. Des moyens, en fin de compte, qui réorganisent les processus cognitifs en interaction appliqués dans la formation en langues étrangères et des processus d' $\mathrm{E} / \mathrm{A}$. Tel que Guichon (2012b, p. 24) le dit «prendre part à des échanges en ligne implique, par conséquent, d'être sensibilisé aux genres et normes propres à la communication médiatisée».

Pour que l'enseignant intègre les TIC, il est essentiel que lui-même fasse l'expérience d'une variété de modalités et d'initiatives dans sa formation. Cela justifie la nécessité d'un plan de formation des enseignants en ligne, dans lequel les outils TIC doivent servir à motiver, stimuler et impliquer dans le processus d'enseigner, faciliter la compréhension et un apprentissage numérique chez l'apprenant. Cette idée d'intégrer les environnements en ligne est conçue pour améliorer la qualité de l'enseignement et s'éloigner progressivement de la réalité dans laquelle les enseignants continuent d'utiliser les ENA comme de simples supports d'apprentissage ou simples transmetteurs d'information. Les études consultées mettent en évidence que la formation à l'éducation virtuelle des enseignants doit se concentrer sur la composante numérique liée à la composante didactique et non au concept instrumental, où les outils numériques doivent être présents de façon active et courante dans les pratiques pédagogiques. La formation des enseignants doit donc être orientée vers l'utilisation et la gestion des outils technologiques ainsi que la formation pédagogique nécessaire pour utiliser et intégrer ces ressources dans les processus d'enseignement-apprentissage.

Notre parcours sur l'ALMT a consisté à connaître, décrire et réfléchir sur la formation initiale des enseignants dans la compétence numérique et savoir comment le processus d'E/A implique les étudiants natifs ou immigrants en technologie. Les possibilités pour améliorer ce processus dans l'enseignement supérieur sont restreintes à l'adaptation à la nouvelle réalité sociale par le biais des TICE. L'intégration du numérique dans le parcours didactique en classe de langues étrangères vise la maîtrise de différents environnements informatiques impliquant une révolution pour les futurs professionnels des langues; si bien, tel que Mangenot (2016) le dit, ces instruments sont au service de l'enseignement, et non le contraire. Enseignants et étudiants doivent acquérir des compétences numériques, être autonomes et s'aider d'outils complémentaires dans le but de s'adapter aux nouvelles pratiques en didactique et au service d'une expérience pédagogique innovante en classe de langues étrangères. Les différentes compétences numériques classifiées et définies par divers organismes internationaux sont mises à la disposition de ces acteurs pour les aider à suivre une progression dans leur formation technologique. Actuellement, les multiples méthodologies d'enseignement (en présentiel, à distance, etc.) et d'apprentissage (dirigé, autoapprentissage, etc.) requièrent une pratique et une actualisation constante par rapport à la familiarisation avec les technologies et au sein des domaines de l'information et l'alphabétisation des données, la communication et la collaboration, la production de contenu numérique, la sécurité et la résolution de problèmes.

Il est nécessaire donc d'intensifier la recherche pour ouvrir la voie et promouvoir l'adoption de nouvelles solutions permettant d'améliorer l'enseignement virtuel. Les institutions et les gouvernements doivent rapidement s'adapter et adopter de nouvelles politiques d'intégration qui garantissent l'équité et l'innovation dans l'enseignement supérieur. Pour garantir une bonne qualité de ce dernier, il est nécessaire d'agir rapidement et de fomenter une actualisation à la hauteur du monde dans lequel ont leur place aussi bien les enseignants que les étudiants. En accord avec cette considération, nous voulons finir notre réflexion en évoquant la sensibilisation aux bonnes pratiques en matière d'enseignement et de recherche et en rappelant le Marco de desarrollo profesional docente del profesorado universitario de Paricio (2018) qui invite précisément

a "pensar la docencia", a revisar críticamente nuestras ideas sobre lo que significa una "buena docencia", a debatirlas y contrastarlas con las conclusiones de la investigación. Este es, necesariamente, el punto de partida para la actualización y mejora de nuestra cultura docente compartida, la base sobre la que construimos, explícita o implícitamente, nuestras prácticas. (Paricio, 2018, p. 14)

\footnotetext{
Quelques institutions consolidées dans le mouvement MOOC et l'enseignement supérieur sont Coursera, Canvas, Udacity, edX, Miríada et UniMOOC (Vázquez et al., 2013).
} 


\section{LISTE DES ABRÉVIATIONS}

ALAO: Acquisition ou apprentissage des langues assisté par ordinateur

ALMT: Apprentissage de langues médiatisé par les technologies

CALL: Computer-assisted language learning

CARAP: Cadre de référence pour les approches plurielles des Langues et des Cultures

CECR: Cadre européen commun de référence pour les langues

CLOM: Cours en ligne ouverts et massifs

CMC: Computer-mediated communication

CMCL: Computer-mediated communication for language learning and teaching

CMO: Communication médiatisée par ordinateur

cMOOC: MOOC connectiviste

DDL: Didactique des langues

DigComp: The digital Competence Framework for Citizens

DLC: Didactique des langues-cultures

E/A: Processus d'enseignement-apprentissage

EAO: Enseignement assisté par ordinateur

EEES: Espace européen de l'enseignement supérieur

ENA: Environnements numériques d'apprentissage

ICT: Information and communication technologies

ICT-CFT: Référentiel de compétences TIC pour les enseignants

LMOOC: Language massive open online courses

LMS: Learning management systems

MOOC: Massive open online course

NOOC: Nano open online massive

SPOC: Small private online course

SPOOC: Self-paced open online course

TIC: Technologies de l'information et de la communication

TICE: Technologies de l'information et de la communication pour l'enseignement

tMOOC: Transfer Massive Open Online Course, MOOC constructiviste

xMOOC: MOOC transmissif

\section{BIBLIOGRAPHIE}

Abadía Valle, Ana Rosa, Concepción Bueno García, María Isabel Ubieto-Artur, M. Dolores Márquez Cebrián, Sarai Sabaté Díaz, Helga Jorba Noguera et Teresa Pagès Costa (2015). Competencias del buen docente universitario. Opinión de los estudiantes. REDU, Revista de Docencia Universitaria, 13(2), 363-390. Https://doi.org/10.4995/redu.2015.5453.

Albero, Brigitte (2003). Autoformation et enseignement supérieur. Paris: Lavoisier.

Albero, Brigitte (2010). Penser le rapport entre formation et objets techniques. Dans Gilles Leclercq et Renáta Varga (éds). Dispositifs de formation et environnements numériques: enjeux pédagogiques et contraintes informatiques (pp. 38-69). Paris: Hermès-Lavoisier.

Albero, Brigitte (2013). Quels enjeux pour les recherches sur les usages du numérique dans l'enseignement supérieur? Distances et Médiations des Savoirs, 1(4). Https://doi.org/10.4000/dms.367.

André, Bernard (dir.) (1989). Autonomie et enseignement-apprentissage des langues étrangères. Paris: Didier/Hatier.

Area, Manuel (2010). ¿Por qué formar en competencias informacionales y digitales en la educación superior? RUSC. Revista de Universidad y Sociedad del Conocimiento, 7(2), 2-5. Http://dx.doi.org/10.7238/rusc.v7i2.976.

Barbot, Marie-José (2001). Les auto-apprentissages. Paris: CLE International.

Barroso Osuna, Julio (2019). Enseñar y aprender en la Red (II): nuevas perspectivas. Dans Sara Robles Ávila et Antonio Moreno Ortiz (éds). Comunicación mediada por ordenador: la lengua, el discurso y la imagen (pp. 93-111). Madrid: Cátedra.

Bates, Tony (2001). Cómo gestionar el cambio tecnológico. Estrategias para los responsables de centros universitarios (trad. de R. Filella Escolà). Barcelona: Gedisa.

Beacco, Jean-Claude et Daniel Luzzati (1999). Apprendre les langues à distance? Formes actuelles et à venir des environnements d'enseignement/apprentissage (EIA) sur support numérique. Le Français dans Le Monde, $\mathrm{n}^{\circ}$ spécial, 31-42. Http://www.academia.edu/attachments/59546061/download_file?s=portfolio.

Beacco, Jean-Claude, M. di Giura, O. Gueye, P. Teusch, et Fr. Tauzeur Sabatelli (2000). Enseigner à distance le français comme langue étrangère: principes pour l'élaboration d'un environnement d'apprentissage numérique et interactif intégré. Actes des deuxièmes entretiens internationaux sur l'Enseignement à distance, tome 2 (pp. 209-216). Poitiers: CNED. 
Cabero Almenara, Julio (2019). Enseñar y aprender en la Red (I): sus aplicaciones institucionales. Dans Sara Robles Ávila et Antonio Moreno Ortiz (éds). Comunicación mediada por ordenador: la lengua, el discurso y la imagen (pp. 75-92). Madrid: Cátedra.

Carretero, Stephanie, R. Vuorikari et Y. Punie (2017). DigComp 2.1. The digital Competence Framework for Citizens with eight proficiency levels and examples of use. Luxembourg: Luxembourg Publication Office of the European Union et JRC Science for policy report. Https://doi.org/10.2760/38842.

Chanier, Thierry (1995). Acquisition des Langues Assistée par Ordinateur (ALAO) [Synthèse présentée pour l'obtention d'une habilitation à diriger des recherches]. Université Blaise Pascal, Clermont-Ferrand, France.

Delgado, Arturo (2003). La enseñanza del francés en el siglo xx: métodos y enfoques. Anales de Filología Francesa, 11, 79-96. Https://revistas.um.es/analesff/article/view/19521.

Demaizière, Françoise (1986). Enseignement Assisté par Ordinateur. Paris: Ophrys.

Demaizière, Françoise et Muriel Grosbois (2014). Numérique et enseignement-apprentissage des langues en Lansad Quand, comment, pourquoi? Alsic. Apprentissage des Langues et Systèmes d'Information et de Communication, 17. https://doi.org/10.4000/alsic.2691.

Develotte, Christine (2010). Réflexions sur les changements induits par le numérique dans l'enseignement et l'apprentissage des langues. Éla. Études de Linguistique Appliquée, 160(4), 445-464. Https://doi.org/10.3917/ela.160.0445.

Dieuzeide, Henri (1994). Les nouvelles technologies: outils d'enseignement. Paris: Nathan.

Fernández, Amparo et Javier Paricio (2019). Entornos de aprendizaje como espacios para la acción, interacción, autorregulación y elaboración personal del conocimiento. Dans Javier Paricio, Amparo Fernández et Idoia Fernández (éds.). Cartografía de la buena docencia. Un Marco para el desarrollo del profesorado basado en la investigación (pp. 197-216). Madrid: Narcea.

Gabarrón Pérez, Ángel, Ana María Pino Rodríguez, Carlos Salvadores Merino et Fernando Trujillo Sáez (2020). Tecnología para la enseñanza y el aprendizaje de lenguas extranjeras. La Enseñanza de lenguas Asistida por Ordenador. Pasado, presente y futuro. Pragmalingüística, 28, 238-254. Https://doi.org/10.25267/Pragmalinguistica.2020.i28.12.

Gisbert, Mercè et Francesc Esteve (2011). Digital Leaners: la competencia digital de los estudiantes universitarios. $L a$ Cuestión Universitaria, 7, 48-59. Http://polired.upm.es/index.php/lacuestionuniversitaria/article/view/3359.

Grosbois, Muriel (2012). Didactique des langues et technologies: de l'EAO aux réseaux sociaux. Paris: PUPS.

Grosbois, Muriel (2015). S'approprier le numérique: réussite ou échec? Pour qui, pourquoi, comment? Recherche et Pratiques Pédagogiques en Langues de Spécialité, Cahiers de l'APLIUT. Https://doi.org/10.4000/apliut.5168.

Guichon, Nicolas (2012a). Vers l'intégration des TIC dans l'enseignement des langues. Paris: Didier.

Guichon, Nicolas (2012b). L'apprentissage des langues médiatisé par les technologies (ALMT) - Étude d'un domaine de recherche émergent à travers les publications de la revue Alsic. Alsic, Apprentissage des Langues et Systèmes d'Information et de Communication, 15(3). Https://doi.org/10.4000/alsic.2539.

Guichon, Nicolas (2015). Quelle transition numérique pour les étudiants internationaux ? Alsic. Apprentissage des Langues et Systèmes d'Information et de Communication, 18(1). Https://doi.org/10.4000/alsic.2793.

Holec, Henri (1989a). Autonomie et apprentissages des langues étrangères. Dans B. André (dir.). Autonomie et enseignement-apprentissage des langues étrangères (pp. 31-33). Paris: Didier/Hatier.

Holec, Henri (1989b). Enseignement et autonomisation. Dans B. André (dir.). Autonomie et enseignement-apprentissage des langues étrangères (pp. 151-154). Paris: Didier/Hatier.

Kern, Richard (2006). La communication médiatisée par ordinateur en langues: recherches et applications récentes aux USA. Le Français dans le Monde, Recherches et Applications, 40, 17-29.

Kern, Richard (2010). Technology and language learning. Dans James Simpson (dir.). The Routledge Handbook of Applied Linguistics (pp. 202-217). New York: Taylor \& Francis.

Lamy, Marie-Noëlle et Regine Hampel (2007). Online communication in language learning and teaching. Basingstoke: Palgrave Macmillan. Https://doi.org/10.1057/9780230592681.

Lebrun, Marcel (2011). Impacts des TIC sur la qualité des apprentissages des étudiants et le développement professionnel des enseignants: vers une approche systémique. STICEF, Sciences et Technologies de l'Information et de la Communication pour l'Éducation et la Formation, 18, 1-20. Https://doi.org/10.3406/stice.2011.1028.

Lévy, Michael (1997). Computer-Assisted Language Learning. Context and Conceptualization. Oxford: Clarendon Paperbacks.

Linard, Monique (2003). Autoformation, éthique et technologies: enjeux et paradoxes de l'autonomie. Dans Brigitte Albero (dir.). Autoformation et enseignement supérieur (pp. 241-264). Paris: Lavoisier.

Mangenot, François (2000). L'intégration des TIC dans une perspective systémique. Les Langues Modernes, 3, 38-44.

Mangenot, François (2003). Tâches et coopération dans deux dispositifs universitaires de formation à distance. Alsic. Apprentissage des Langues et Systèmes d'Information et de Communication, 6(1), 109-125. Https://doi.org/10.4000/ alsic. 2167.

Mangenot, François (2011). Introduction. Du e-learning aux interactions pédagogiques en ligne. Dans Elke Nissen, Françoise Poyet et Thierry Soubrié (dir.). Interagir et apprendre en ligne (pp. 7-19). Grenoble, Ellug: Université Stendhal. Https://doi.org/10.4000/books.ugaeditions.1174.

Mangenot, François (2013). Les échanges en ligne comme secteur de pratiques et de recherches en ALAO: quelles problématiques, quelles évolutions? Cahiers de l'ILOB, 5, 3-21. Https://doi.org/10.18192/olbiwp.v5i0.1114. 
Mangenot, François (2016). Le numérique entre effets de mode et réelle innovation. Dans Anthippi Potolia et Diana Lemay (dirs.). Enseignement / apprentissage des langues et pratiques numériques émergentes. Paris: Éditions des Archives Contemporaines.

Mangenot, François (2017). Formation en ligne et MOOC: Apprendre et se former en langue avec le numérique. Paris: Hachette.

Massou, Luc (2017). Chapitre 10. Médiatisation numérique des ressources pédagogiques: usages, allants de soi et changement. Dans Nathalie Lavielle-Gutnik et Luc Massou (éds.). Enseigner à l'université avec le numérique Savoirs, ressources, médiations (pp. 181-198). Louvain-la-Neuve: De Boeck Supérieur. Https://doi.org/10.3917/dbu. masso.2017.01.0181.

Montero Curiel, Ma Luisa (2019). La desincronización de la comunicación síncrona. Dans Sara Robles Ávila et Antonio Moreno Ortiz (éds). Comunicación mediada por ordenador: la Lengua, el discurso y la imagen (pp. 162-181). Madrid: Cátedra.

Moulden, Harvey (1989). Apprentissage autodirigé: compte rendu d'apprentissage 1878-1983. Dans Bernard André (dir.). Autonomie et enseignement-apprentissage des langues étrangères (pp. 41-50). Paris: Didier/Hatier.

Muñoz Moreno, José Luis et José Peirats (2016). Retos de la tecnología educativa: formación del profesorado y materiales curriculares digitales. Dans C. Suárez, D. Palomares et D. Martín (coords.). Retos de la educación en tiempos de cambio (pp. 189-213). Valencia: Tirant lo Blanch.

Ollivier, Christian et Laurent Puren (2013). L'ouverture au-delà des murs de la classe: du fantasme à la réalité. Le Français dans le Monde. Recherches et Applications. Mutations Technologiques, Nouvelles Pratiques Sociales et Didactique des Langues, 54, 16-27.

Ollivier, Christian et Projet e-lang (2018). Littératie numérique et approche socio-interactionnelle pour l'enseignementapprentissage des langues. Strasbourg: Éditions du Conseil de l'Europe. Https://www.ecml.at/Portals/1/5MTP/ Ollivier/e-lang\%20FR.pdf.

Organisation des Nations Unies pour l'éducation, la science et la culture (2012). TIC UNESCO: un référentiel de compétences pour les enseignants. Paris: UNESCO et Microsoft 2011. Https://unesdoc.unesco.org/ark:/48223/ pf0000216910.

Organisation des Nations Unies pour l'éducation, la science et la culture (2018). Référentiel UNESCO de compétences TIC pour les enseignants. Paris: UNESCO. Https://unesdoc.unesco.org/ark:/48223/pf0000368966.

Paricio, Javier (2018). Marco de desarrollo profesional del profesorado universitario. Planteamiento general y dimensiones. Zaragoza: REDU (Red Estatal de Docencia Universitaria). Https://red-u.org/wp-content/uploads/2018/06/ MarcoREDU_Doc1_PlanteamGral_1.pdf.

Pérès, Lucile (2013). L'évolution des pratiques dans l'enseignement des langues vivantes étrangères à l'école primaire est-elle en phase avec l'évolution des approches de cet enseignement? [Mémoire fin de master, École Supérieure du Professorat et 1'Éducation de Toulouse Midi-Pyrénées, France]. Https://dumas.ccsd.cnrs.fr/dumas-00976958/ document.

Pérez Cervantes, Mónica Luz et Anuar Francisco Saker (2016). Importancia del uso de las plataformas virtuales en la formación superior para favorecer el cambio de actitud hacia las TIC. Estudio de caso: Universidad del Magdalena, Colombia. Revista Iberoamericana de Evaluación Educativa, 6(1). Https://revistas.uam.es/riee/ article/view/3847.

Prensky,Marc(2001). Digitalnatives, digitalimmigrants. OntheHorizon,9(5).Https://doi.org/10.1108/10748120110424816.

Puren, Christian (2012). Histoire des méthodologies de l'enseignement des langues. Paris: Nathan-CLE International. Https:/www.christianpuren.com/mes-travaux/1988a/.

Puren, Christian (2013). Technologies éducatives et perspective actionnelle: quel avenir pour les manuels de langue? Le Français dans le Monde, 54, 122-130. Https://www.christianpuren.com/mes-travaux/2013k/.

Puren, Christian (2019). L'interculturel, une composante parmi d'autres de la compétence culturelle. Neofilolog, 52(2), 213-226. Https://doi.org/10.14746/n.2019.52.2.2.

Rivens Mompean, Annick et Martine Eisenbeis (2009). Autoformation en langues: quel guidage pour l'autonomisation? Recherches en Didactique des Langues et des Cultures, 6(1). Https://doi.org/10.4000/rdlc.2204.

Rodríguez Correa, Marisol et Mercedes González Sanmamed (2013). La gestión del cambio institucional en las universidades a través de las TIC. REDU: Revista de Docencia Universitaria, 11(3), 363-381. Https://doi.org/10.4995/ redu.2013.5533.

Schaeffer-Lacroix, Eva (2020). Les corpus web à travers le prisme de 1'ALMT. Corpus, 20. Https://doi.org/10.4000/ corpus. 4579 .

Soubrié, Thierry (2020). Penser le numérique: orientations pédagogiques et préoccupations de recherche en didactique des langues. Alsic. Apprentissage des Langues et Systèmes d'Information et de Communication, 23. Https://doi. org/10.4000/alsic.4687.

Tremblay, Nicole Anne (2003). L'autoformation. Pour apprendre autrement. Montréal: Presses de l'Université de Montréal. Https://doi.org/10.4000/books.pum.10719.

Trujillo Sáez, Fernando et Francisco Raso Sánchez (2010). Formación inicial docente y competencia digital en la convergencia europea (EEES). Enseñanza \& Teaching, 28(1), 49-77. Https://revistas.usal.es/index.php/0212-5374/ article/view/7481. 
Trujillo Sáez, Fernando, Carlos Salvadores Merino et Ángel Gabarrón Pérez (2019). Tecnología para la enseñanza y el aprendizaje de lenguas extranjeras: revisión de la literatura. RIED: Revista Iberoamericana de Educación a Distancia, 22(1), 153-169. Https://doi.org/10.5944/ried.22.1.22257.

Vázquez Cano, Esteban, Eloy López Meneses et Julio Barroso Osuna (2013). El futuro de los MOOC. Retos de la formación on line, masiva y abierta. Madrid: Síntesis.

Vuorikari, Rina, Y. Punie, S. Carretero et L. Van den Brande (2016). DigComp 2.0: The Digital Competence Framework for Citizens. Update Phase 1: The Conceptual Reference Model. Luxembourg: Luxembourg Publication Office of the European Union \& JRC Science for policy report. Https://doi.org/10.2791/11517. 